\title{
RINGS WITH A POLYNOMIAL IDENTITY
}

\section{IRVING KAPLANSKY}

1. Introduction. In connection with his investigation of projective planes, M. Hall [2, Theorem 6.2 $]^{1}$ proved the following theorem: a division ring $D$ in which the identity

$$
(x y-y x)^{2} z=z(x y-y x)^{2}
$$

holds is either a field or a (generalized) quaternion algebra over its center $F$. In particular, $D$ is finite-dimensional over $F$, something not assumed a priori. The main result (\$2) in the present paper is the following: if $D$ satisfies any polynomial identity it is finite-dimensional over $F$. There are connections with other problems which we note in $\S \S 3,4$.

2. Proof of finite-dimensionality. Let $A$ be an algebra (no assumption of finite order) over a field $F$. We denote by $F\left[x_{1}, \cdots, x_{r}\right]$ the free algebra generated by $r$ indeterminates over $F$. We say that $A$ satisfies a polynomial identity if there exists a nonzero element $f$ in $F\left[x_{1}, \cdots, x_{r}\right]$ such that $f\left(a_{1}, \cdots, a_{r}\right)=0$ for all $a_{i}$ in $A$.

Lemma $1 .^{2}$ If $A$ satisfies any polynomial identity, then it satisfies a polynomial identity in two variables.

Proof. Suppose $A$ satisfies the equation $f\left(x_{1}, \cdots, x_{r}\right)=0$. Replacing $x_{i}$ by $u^{i} v$ we obtain the equation $g(u, v)=0$, with $g$ a polynomial which is not identically zero.

Lemma 2. If $A$ satisfies any polynomial identity, it satisfies a polynomial identity which is linear in each variable.

Proof. Suppose $A$ satisfies $f\left(x_{1}, \cdots, x_{r}\right)=0$ and that $f$ is not linear in $x_{1}$. Then

$$
f\left(y+z, x_{2}, \cdots, x_{r}\right)-f\left(y, x_{2}, \cdots, x_{r}\right)-f\left(z, x_{2}, \cdots, x_{r}\right)=0
$$

is satisfied by $A$. This is a polynomial (in $r+1$ variables), not identically zero, and with degree in $y$ and $z$ lower than the degree of $f$ in $x_{1}$. By successive steps of this kind we reach a polynomial linear in all variables.

Presented to the Society, September 3, 1947; received by the editors August 20, 1947.

1 Numbers in brackets refer to the bibliography at the end of the paper.

${ }^{2}$ Cf. [7, Satz 2]. 
Lemma 3. Suppose that $A$ satisfies an identity $g=0$ where $g$ is linear in all its variables, and let $K$ be a field over $F$. Then the Kronecker product $A \times K$ also satisfies $g=0$.

Proof. We have to show that $g\left(x_{1}, \cdots, x_{r}\right)$ vanishes when the $x$ 's are replaced by elements $\sum a_{i} k_{i}$ of $A \times K\left(a_{i} \in A, k_{i} \in K\right)$. This follows from the linearity of $g$ and the fact that the $k$ 's commute with everything.

Lemma $4 .^{3}$ If $A$ satisfies an identity $f=0$ which is of degree not greater than $n$ in each of its variables, and if $F$ has at least $n+1$ elements, then $A$ satisfies an identity which is homogeneous in each of its variables.

Proof. We write $f=\sum f_{i}$ where $f_{i}$ is of degree $i$ in $x_{1}$. Replacing $x_{1}$ by $\lambda x_{1}$ where $\lambda \in F$, we find $\sum \lambda{ }^{i} f_{i}=0$. We do this for $n$ different nonzero scalars. Using the resulting Vandermonde determinant, we obtain $f_{i}=0$. We repeat this procedure with each of the variables.

LEMma 5. If $f(x, y)$ is any nonzero element of $F[x, y]$, we can find two matrices $a, b$ with elements in $F$ such that $f(a, b) \neq 0$.

Proof. A field of degree $k$ over $F$ may be represented by $k \times k$ matrices over $F$. Thus by taking matrices split into suitable smaller blocks, we may arrange for a scalar field of any desired (finite) size.

Suppose that on the contrary $f$ vanishes for all matrices with elements in $F$. Then by Lemma 4 we may pass to a polynomial $g(x, y)$ which also vanishes for any matrices over $F$, and which is homogeneous in $y$, say of degree $t$. Take $a$ to be a diagonal matrix: $a=\operatorname{diag}\left(u_{1}, \cdots, u_{n}\right)$, with $n$ greater than the degree of $f$, and the $u$ 's as yet undefined. For $b$ we choose an $n$ by $n$ matrix which permutes the elements of a diagonal matrix cyclically. Thus

$$
b^{i} a b^{-i}=\operatorname{diag}\left(u_{i+1}, u_{i+2}, \cdots, u_{n}, u_{1}, \cdots, u_{i}\right) .
$$

We consider $g(a, b) b^{-t}$. Using the relation (2), we systematically push $b$ to the right of $a$, and every such operation induces a cyclic permutation. Corresponding to the typical monomial $x^{i} y^{j} x^{k} y^{l} x^{m} \ldots$ in $g$, we obtain the term

$$
\left(u_{1}\right)^{i}\left(u_{1+j}\right)^{k}\left(u_{1+j+l}\right)^{m} \ldots
$$

in the upper left corner of $g(a, b) b^{-t}$. Moreover given any such term we can unambiguously reconstruct the monomial from which it arose (we use here the fact that $n$ exceeds the degree of $g$, so that there is no overlapping of the $u$ 's). Thus the upper left entry of $g(a, b) b^{-t}$ is a

\footnotetext{
${ }^{3}$ Cf. [7, Satz 1].
} 
polynomial $h\left(u_{1}, \cdots, u_{n}\right)$ (that is to say, an ordinary commutative polynomial), which is not identically zero. By enlarging the field of scalars, if again necessary, we can find values of the $u$ 's which make $h$ different from 0 . For this choice we have $g(a, b) \neq 0$, a contradiction.

We prove the main theorem of the paper not only for division rings, but more generally for primitive algebras in the sense of Jacobson.

Theorem 1. A primitive algebra satisfying a polynomial identity is finite-dimensional over its center.

Proof. First we assert that $A$ is a matrix algebra of finite order over a division algebra. For if not we would have $e^{4}$ that for every $k$, $A$ has a sub-algebra homomorphic to $D_{k}$ where $D$ is a division algebra over $F$, and $D_{k}$ is the algebra of matrices of order $k$ over $D$. The polynomial identity satisfied by $A$ is inherited by $D_{k}$ for all $k$, and therefore holds for all matrices with coefficients in $F$. By Lemma 1 we may assume that the polynomial in question has two variables, and this contradicts Lemma 5 .

The problem is thus reduced to the case where $A$ is a division algebra, say with center $Z$. By Zorn's lemma, select a maximal subfield $K$ of $A$, and form the Kronecker product $A \times K$ over $Z$. By a theorem of Nakayama and Azumaya [7], $A \times K$ is a dense algebra of linear transformations in a vector space over $K$. By Lemma $2, A$ has a linear polynomial identity, and by Lemma 3 this identity survives in $A \times K$. By Lemma 1 we pass to an identity in two variables in $A \times K$. Repetition of the argument of the preceding paragraph shows that $A \times K$ is finite-dimensional over $K$, hence $A$ is finite-dimensional over $Z$.

The following further result is virtually a restatement of Lemma 5.

THEOREM 2. The free algebra in any number of indeterminates has a complete set of finite-dimensional representations.

PRoof. Let $f\left(x_{1}, \cdots, x_{r}\right)$ be an element in the free algebra. We make the replacement $x_{i}=u^{i} v$ of Lemma 1 . For the resulting polynomial $g(u, v)$, we can find, by Lemma 5 , matrices $a$ and $b$ such that $g(a, b) \neq 0$. The mapping induced by sending $x_{i}$ into $a^{i} b(i=1, \cdots, r)$ and all other $x$ 's into 0 is a homomorphism of the free algebra into the algebra generated by $a$ and $b$, and in this representation $f\left(x_{1}, \cdots, x_{r}\right)$ is not sent into 0 .

3. Algebraic algebras. It is true conversely that any algebra of finite order satisfies a polynomial identity. For algebras over the real numbers, this was shown by Wagner [8, pp. 531-532]; his identity

- [3, Th. 3]. Jacobson has since shown that a unit element is not necessary for this theorem (oral communication). 
for $n$ by $n$ matrices has degree $\left(n^{2}-n+2\right)\left(n^{2}-n+4\right) / 4$. E. R. Kolchin has remarked that any algebra of order $n$ satisfies an identity of degree $n+1$ :

$$
\sum \pm x_{1} x_{2} \cdots x_{n+1}=0
$$

where the sign is positive or negative according as the permutation is even or odd. Incidentally the existence of these identities furnishes a brief proof of Malcev's result [6, Theorem 9] that the free algebra in two or more indeterminates cannot be faithfully represented by matrices of finite order.

A wider class of algebras than those of finite order is covered by the following result. ${ }^{5}$

Theorem 3. An algebraic algebra of bounded degree satisfies a polynomial identity.

PRoof. To avoid complications of notation, we give the proof for the case where the degrees of the elements are bounded by 3 . Thus every element $x$ satisfies an equation

$$
x^{3}+\alpha x^{2}+\beta x=0
$$

where $\alpha, \beta$ are scalars which of course depend on $x$. (We do not assume a unit element, and so no constant appears in (3).) We take the commutator with $y$, obtaining

$$
\left[x^{3}, y\right]+\alpha\left[x^{2}, y\right]+\beta[x, y]=0
$$

where $[x, y]$ denotes $x y-y x$. Then we take the commutator with $[x, y]$ and finally with $\left[[x, y],\left[x^{2}, y\right]\right]$. The result is:

$$
\left[\left[[x, y],\left[x^{2}, y\right]\right],\left[[x, y],\left[x^{3}, y\right]\right]\right]=0,
$$

a polynomial of degree 11 . The polynomial moreover is not identically zero, since for example there is only one term

$$
x y x^{2} y x y x^{3} y \text {. }
$$

In the general case where the degree is bounded by $n$, we get an identity of degree $2^{n+1}-2^{n-1}-1$. The case $n=2$ yields an identity equivalent to (1), with $z$ replaced by $x$.

Combining Theorems 1 and 3, we obtain a new proof of a theorem due to Jacobson [3, Theorems 5 and 7].

Theorem 4. A primitive algebraic algebra of bounded degree is finitedimensional over its center.

\footnotetext{
I I am indebted to Prof. Jacobson for Theorem 3 and its proof.
} 
An outstanding question in the theory of algebraic algebras is Kurosch's analogue of Burnside's problem: is an algebraic algebra necessarily locally finite? ${ }^{6}$ Affirmative answers have been contributed in two cases: (1) algebraic algebras of bounded degree [3, 4, and 5], and (2) representable ${ }^{7}$ algebraic algebras [6]. A third (trivial) instance where the answer is affirmative is the commutative case. A hypothesis that is weaker than any of these is the assumption of a polynomial identity. It thus seems natural to try Kurosch's problem next for algebraic algebras with a polynomial identity. We shall now contribute an affirmative answer for the case of a nil algebra; in the light of $[3$, Theorem 15] the question is thus reduced to the semi-simple case.

Theorem 5. A nil algebra satisfying a polynomial identity is locally finite.

Proof. We use the terminology and results of Levitzki (cf. [5] and the references given there). Exactly as in [5] we reduce to the case where $A$ is semi-regular. We choose an element $a \neq 0$ such that $a^{2}=0$. The algebra $A a$ is also semi-regular. We convert our identity into one, say $f\left(x_{1}, \cdots, x_{r}\right)=0$, which is linear in each variable (Lemma 2). Suppose the variable $x_{1}$ actually appears first in at least one of the monomials comprising $f$. We gather all such terms and write

$$
f\left(x_{1}, \cdots, x_{r}\right)=x_{1} g\left(x_{2}, \cdots, x_{r}\right)+h\left(x_{1}, \cdots, x_{r}\right) .
$$

Each monomial of $h$ has a factor $x_{1}$ which appears later than the first term. It follows that if we substitute $a$ for $x_{1}$, and any elements of $A a$ for $x_{2}, \cdots, x_{r}$, we introduce a factor $a^{2}$ in each term of $h$ and thus make $h$ vanish. Thus $a g\left(x_{2}, \cdots, x_{r}\right)$ must also vanish for $x_{2}, \cdots, x_{r}$ in $A a$. This makes $g$ a right annihilator of $A a$. Since $A a$ is semi-regular it has no right annihilator except 0 . Hence $A a$ satisfies the identity $g=0$. By induction on the degree of the identity we have that $A a$ is locally finite, which contradicts its semi-regularity.

4. Further remarks. (a) Wagner's main theorem in [8] asserts that an ordered algebra over the reals satisfying a polynomial identity is necessarily commutative. Our results furnish a short proof of a special case: any ordered primitive algebra satisfying a polynomial identity is commutative. This is an immediate consequence of Theorem

\footnotetext{
${ }^{6}$ An algebra is locally finite if every finitely generated sub-algebra is of finite order.

"An algebra is representable if it is isomorphic to a ring of matrices of finite order over some extension field.
} 
1 and Albert's theorem [1] to the effect that any ordered algebra of finite order is a field.

(b) The argument of Theorem 1 can be made to yield an explicit upper bound for the order of the algebra over its center, in terms of the degree and number of variables of the identity; the bound however is far too generous. In a special case like Hall's theorem there is no difficulty in getting the precise bound. Specifically it is only necessary to produce 3 by 3 matrices violating the linearized form of (1). One may in this way also verify the following result: a semi-simple algebra satisfying a polynomial identity of degree not greater than 3 is commutative.

(c) We have for simplicity given all the results in this paper for algebras, but they may be extended to rings as follows. Assume that the polynomials in question have as coefficients operators $\alpha$ such that $\alpha x=0$ implies $x=0$. It is to be observed that this holds in particular if the coefficients are \pm 1 . Thus we may assert that any primitive ring satisfying (1) is an algebra of finite order; and Theorem 5 thus extended subsumes Levitzki's theorem [5] that a finitely generated nil-ring of bounded index is nilpotent.

Added in proof (May 1, 1948). I am indebted to Dr. HarishChandra for the following brief proof of Lemma 5. Let $n$ be the degree of $f(x, y)$ and $I$ the ideal $F[x, y]$ generated by monomials of degree not less than $n+1$. Then $F[x, y] / I$ is an algebra, which can be faithfully represented by matrices since it is of finite order. This gives us matrices for which $f \neq 0$.

\section{BIBLIOGRAPHY}

1. A. A. Albert, On ordered algebras, Bull. Amer. Math. Soc. vol. 46 (1940) pp. 521-522.

2. M. Hall, Projective planes, Trans. Amer. Math. Soc. vol. 54 (1943) pp. 229-277.

3. N. Jacobson, Structure theory for algebraic algebras of bounded degree, Ann. of Math. (2) vol. 46 (1945) pp. 695-707.

4. I. Kaplansky, On a problem of Kurosch and Jacobson, Bull. Amer. Math. Soc. vol. 52 (1946) pp. 496-500.

5. J. Levitzki, On a problem of A. Kurosch, Bull. Amer. Math. Soc. vol. 52 (1946) pp. $1033-1035$.

6. A. Malcev, On the representations of infinite algebras, Rec. Math. (Mat. Sbornik) N. S. vol. 13 (1943) pp. 263-286 (Russian with English summary).

7. T. Nakayama and G. Azumaya, Über einfache distributive Systeme unendlicher Range II, Proc. Imp. Acad. Tokyo vol. 20 (1944) pp. 348-352.

8. W. Wagner, Über die Grundlagen der projektiven Geometrie und allgemeine Zahlensysteme, Math. Ann. vol. 113 (1937) pp. 528-567.

University of Chicago 